\title{
Laser Processing Gold Interconnectors for Anti-Erosion Protection from Atomic Oxygen in Space
}

\author{
Hongjie Wang ${ }^{1, a^{*}}$, Chang Liu ${ }^{2, b}$, Xuefeng Lv ${ }^{3, c}$, Wenzhe Lv ${ }^{4, d}$, Xiaokang \\ Huang ${ }^{5,}$ and Jinfu Gao, ${ }^{6, f}$ \\ $1,2,3,4,5,6$ School of physics, Nankai University, Tianjin 300071, China \\ àwang_hj@nankai.edu.cn,b844755001@qq.com ,'363052564@qq.com,d1282631536@qq.com, \\ e245659554@qq.com, \\ fgao867873513@sina.com
}

\begin{abstract}
Keywords: Laser Machining, Ytterbium-Doped Fiber Laser, Gold Interconnector, Anti-Erosion.
Abstract. Under processing condition that were the average power of $20 \mathrm{~W}$, repetition frequency of 2 $\mathrm{kHz}$, pulse width of $80 \mathrm{~ns}$, spot diameter less than $20 \mu \mathrm{m}$, cutting speed of $10 \mathrm{~mm} / \mathrm{s}$, we succeeded in making $300 \mu \mathrm{m}$ thick gold interconnectors for solar cell panels, by utilizing an optical fiber laser with high power density. These interconnectors could effectively prevent the oxidation corrosion from atomic oxygen in space, meanwhile reduce ohm-contact and series resistance of electrodes. Therefore, both the performance and stability of solar cell could be significantly improved.
\end{abstract}

\section{Introduction}

The effects of atomic oxygen (monatomic oxygen) bombardment are extensively observed on spacecraft operated at a fast speed in low Earth orbit. Atmospheric atomic oxygen will cause performance, especially photoelectrical and thermodynamic, degradation of the key components, even threaten the normal flight of spacecraft. For example, when a silver interconnector that is widely used in the solar cell panels on spacecraft encounters with atomic oxygen, the strong oxidation corrosive effect will occur. And this will lead to an increase in ohm-contact and series resistance of electrodes, so that the output power will decrease significantly. Besides, due to the formation of thick oxidation layers, the addition weight may drag silver foil off the panel body and lead to the dysfunction of the entire power system.

To avoid the erosion hazard from the space atomic oxygen, evaporating of the organic protective film on the key component is only a remedial method. For the solar cell arrays, substituting the silver foil interconnectors with gold ones is the most direct, effective and feasible scheme [1-3]. To this end, we utilized a high purity of $99.99 \%$ gold foil to make interconnectors in solar cell, which can effectively prevent the oxidation corrosion action from the atomic oxygen. In addition, the high pure gold possess lower resistivity and better stability, which are beneficial to improving the output power and the photoelectric conversion efficiency. However, the reflectivity of gold foil at the near infrared wavelength excesses $90 \%$. Little absorption in this band makes laser processing to this kind of shiny metals extremely difficult. As reported in recent literature, American Applied Material Company first used UV laser with an average power of $3 \mathrm{~W}$ and wavelength of $266 \mathrm{~nm}$, and directly realized the precision machining and marking on thin metal films [4]. In China, Jianhua Yang from Beijing University of Technology also achieved precision laser machining on $100 \mu \mathrm{m}$ copper films by applying a UV laser with maximum output power of $10 \mathrm{~W}$ and the wavelength of $355 \mathrm{~nm}$, under nitrogen-purged low pressure environment [5]. At the same time, Maohua Jiang from the same university used a pulsed ultraviolet laser with repetition frequency of $2.5 \mathrm{kHz}$, and have proceeded sintering experiment on shiny metals [6]. The results show that UV laser with larger photon energy is easier to be absorbed by metal atoms. However, the output power of UV laser is generally small, which cannot meet the requirement of larger scale and deeper machining in industrial processes.

Therefore, in this paper, we utilized an optical fiber laser which possesses a high beam quality and high power density. By optimizing the laser cavity and the laser transfer mode, we have demonstrated precision machining on $300 \mu \mathrm{m}$ thick gold foil with near-infrared (NIR) laser and succeeded in making gold interconnectors for solar cell panels. As fabricated interconnectors are expected to 
efficiently prevent the oxidation corrosion from atmospheric atomic oxygen, and improve the performance and stability of power supply system, our experiment results possess important research significance and application value in out-space solar cell panel studies.

\section{Absorption Theory}

Based on the Finel formula in materials science, Chen Jun from Zhejiang University calculated the approximate absorption rate of free electron on metal surface is as eq. 1 [7]:

$\alpha=0.1457,-,(\rho /-\lambda)^{\wedge} 0.5$.

Here $\alpha$ is the metal absorption rate, $\rho$ is the metal resistivity, $\lambda$ is the laser wavelength. From the above equation, we can see that the metal absorption rate and the square root of laser wavelength is into inverse proportional relationship. After analysis, we are aware that the photon energy at short wavelength (UV range) is larger and comparable to the free energy of electron at metal surface. Thereby, laser beams in this wave range are easier to be absorbed by interacting with free electrons. But at long wavelength (NIR range), single photon energy is relatively smaller and different from the free electron energy. Especially when the laser power density is also very low, such as $10^{4} \sim 10^{6}$ $\mathrm{W} / \mathrm{cm}^{2}$, the metal surface can fully reflect NIR laser beams. Fig. 1 gives several shiny metal's absorption curves [8]. This diagram also shown that the shiny metal's absorption rate is very low at long wavelength, but as the wavelength becomes shorter, the absorption rate will increase rapidly. For gold and silver, the absorption rate at $1064 \mathrm{~nm}$ is less than 10\%, when the wavelength is shorter than $400 \mathrm{~nm}$, the gold and silver material's absorption rate will increase rapidly. That is to say, in the ultraviolet and deep ultraviolet region, the gold and silver material's absorption rate is relatively high. On the other hand, the absorption rate at $266 \mathrm{~nm}$ can reach $70 \%$. Theoretically, UV and DUV beam should be preferred in shiny metal laser machining. However, for such short wavelength lasers, due to the nowadays technical limitations, their output power is generally low, large-scale and deep laser processing is still incapable.

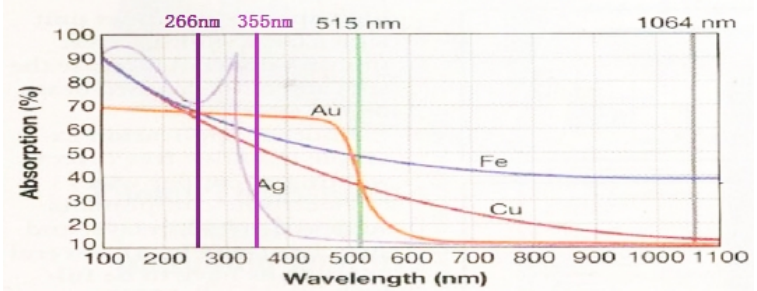

Figure 1. Absorption curve of several common shiny metals.

It's known that metal to laser's absorption coefficient depends primarily on three factors, which are wavelength, power density and temperature, among them, the effect of temperature factor is very small, and changing the temperature of work piece is also difficult, which requires installing a temperature control furnace in the processing platform, so, it is not straight forward to raise the absorption rate by changing the temperature. While utilizing laser with short wavelength is the preferred method, we have already discussed this aspect above. Now, we will mainly discuss about the effect of laser power density. Usually, when the power density reached $10^{9} \sim 10^{11} \mathrm{~W} / \mathrm{cm}^{2}$, the laser machining process can be realized. While the power density is relatively small, such as in the $10^{4} \sim 10^{6}$ $\mathrm{W} / \mathrm{cm}^{2}$ range, the effect of laser irradiation is similar as annealing process, in which only surface modification, surface alloying or welding process can occur. The photon energies are converted to heat energies by work piece absorption, and the temperature of work piece is raised quickly up to the melting point, melting is dominant during this low temperature laser processing. When the laser power density reached $10^{9} \mathrm{~W} / \mathrm{cm}^{2}$ or more, it has already exceeded the threshold power that can induce vaporization of gold and silver, and when laser irradiate on the metal surface, the vaporization and the formation of plasma will occur, this kind of phenomenon is essential in laser drilling, laser cutting, which are removal type of processing, the machined object is directly evaporated into steam from solid, which belongs to the high temperature laser processing, in this case, lasers behave in the 
form of electromagnetic waves and strong interaction with free electrons on metal surface. In fact, it is that metal plasma excited by high brightness and high power density laser has realized the machining of shiny metals, this is just the underline mechanism of high brightness and high power density laser direct etching of shiny metal, which also plays the theoretical basis of our experiment.

\section{Experiment Section}

In experiment, we utilized an ytterbium-doped fiber laser, which possess good beam quality and high power density, with center wavelength at $1060 \mathrm{~nm}$. At the first, we observed multiple laser modes in a lattice distribution, therefore the laser energy is not concentrated. By optimizing the laser operation parameters, we discovered that laser field distribution is mainly affected by the repetition frequency. When we applied the different repetition frequency, following Fig. 2 (a) and (b) shown different laser field distribution, with lower repetition frequency, the number of dot matrix of laser field gradually reduced, when the repetition rate is only $2 \mathrm{kHz}$, that fiber laser can achieve single transverse mode output, and the spot can be focused to less than $20 \mu \mathrm{m}$. As shown in Fig. 2(c), when laser was operated with multimode output, its energy was dispersed, so that the etching result was not ideal. As shown in Fig. 2(d), when laser was operated with single mode output, laser spot diameter could be focused into $20 \mu \mathrm{m}$ or smaller, therefore, laser energy was highly concentrated, so that we could realize direct etching of gold foil interconnector, even with NIR laser. Our optimized experimental condition is as the average power of $20 \mathrm{~W}$, repeat frequency of $2 \mathrm{kHz}$, pulse width of 80 ns, spot diameter of $20 \mu \mathrm{m}$, laser cutting speed at $10 \mathrm{~mm} / \mathrm{s}$, and the thickness of gold foil is $300 \mu \mathrm{m}$, the foil is fresh without any pretreatment. As shown in Fig. 3 is that we have fabricated the gold interconnectors successfully, which meet the process requirements for solar cell panel in out-space.

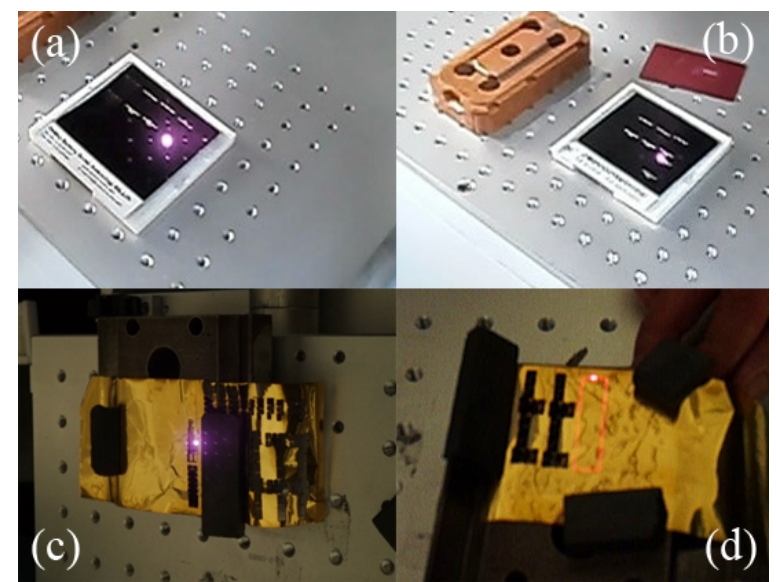

Figure 2. Photo of laser field distribution at repetition Frequency of $20 \mathrm{KHz}$ (a) and $2 \mathrm{KHz}$ (b), Photo of etching gold foil with multimode laser (c) and single mode laser (d).

After optimized, focused laser spot diameter is only $20 \mu \mathrm{m}$, pulse width is $80 \mathrm{~ns}$, the improvement of these parameters greatly increased the laser power density, and after calculation, the actual density is $1.592 \times 10^{9} \mathrm{~W} / \mathrm{cm}^{2}$, which has reached the threshold of high power laser processing, so that the free electrons on metal surface produced plasma lasing, thus to realize a strong absorption of shiny metal at near infrared wavelength. In addition, in the process of high power laser processing, we also observed blue fluorescence emission at the molten part of samples, this may come from two photon generation. Also it is worth to mention that, when the power density is larger than $10^{11} \mathrm{~W} / \mathrm{cm}^{2}$, the resulting in plasma excitation is too intense, it cause surface metal atom splashing phenomenon, which leads to a rough cutting edge and affects the pattern quality. 


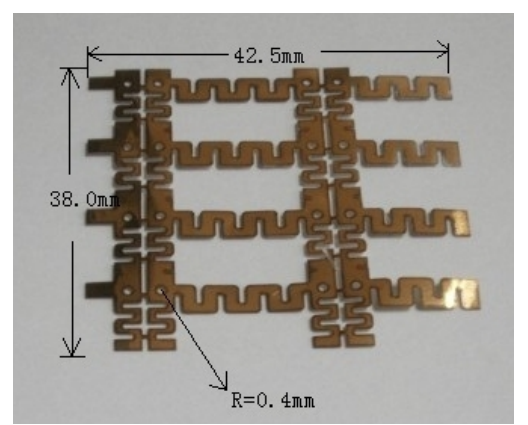

Figure 3. Gold interconnectors by laser cutting.

\section{Conclusion}

In conclusion, to process small devices on shiny metal films, UV or deep DUV lasers with high photon energies are preferred to realize direct laser precision machining. But to fulfill large-scale and deep laser patterning, UV and DUV lasers have been limited output power, which are incapable. Therefore, in this paper, we demonstrated precision machining with high brightness and high power density fiber laser operated at NIR wavelength. By optimizing laser cavity and laser pattern, laser output with a single transverse mode has been achieved and the laser power density has been reached $10^{9} \mathrm{~W} / \mathrm{cm}^{2}$ above the threshold of laser ablation. Gold interconnectors for solar cell panel have been successfully fabricated under the optimized condition.

\section{Acknowledges}

This work was supported by the National Science Fund for Talent Training in Basic Sciences under Grant No. J1103208.

\section{References}

[1] J. Tong, X. Liu, C. Zhang, S. Xiao, S. Duo, and M. Li, Spacecraft Environment Engineering, 2009, 26(1): 1-5(in Chinese).

[2] L. Guo, L. Jiang, T. Li, and X. Liu, Spacecraft Environment Engineering , 2010, 27(4): 428-436(in Chinese).

[3] Z. Wang, Y. Lu, Y. Feng, Y. Wang, and X. Su, Vacuum And Cryogenics, 2004, 10(3): 152-159(in Chinese).

[4] O. Akiyoshi, H. Kenji, H. Koyo, N. Yoshiyuki, and T. Yu, Japanese Journal of Applied Physics, 2009, 48(7): 1201-1205.

[5] J. Yang, J. Chen, and T. Zuo, Chinese Journal of Lasers, 2006, 33(sup.): 423-426.

[6] M. Jiang, L. Zhai, and J.Chen, Microfabrication Technology, 2008, (4): 27-31(in Chinese).

[7] J. Chen, Q. Zhang, J. Yao, and J. Fu, Jour. of Applied Optics, 2008, 29(5): 793-798.

[8] J. Powell, S.O. Mashikhi, A.F.H. Kaplan, and K.T. Voisey, Optics and Lasers in Engineering, 2011, 49: 1069-1075. 